\title{
Dissecting Policy Designs: An Application of the Institutional Grammar Tool
}

\author{
Saba Siddiki, Christopher M. Weible, Xavier Basurto, and John Calanni
}

\begin{abstract}
An enduring challenge for the policy and political sciences is valid and reliable depiction of policy designs. One emerging approach for dissecting policy designs is the application of Sue Crawford and Elinor Ostrom's institutional grammar tool. The grammar tool offers a method to identify, systematically, the core elements that comprise policies, including target audiences, expected patterns of behavior, and formal modes of sanctioning for noncompliance. This article provides three contributions to the study of policy designs by developing and applying the institutional grammar tool. First, we provide revised guidelines for applying the institutional grammar tool to the study of policy design. Second, an additional component to the grammar, called the oBject, is introduced. Third, we apply the modified grammar tool to four policies that shape Colorado State Aquaculture to demonstrate its effectiveness and utility in illuminating institutional linkages across levels of analysis. The conclusion summarizes the contributions of the article as well as points to future research and applications of the institutional grammar tool.
\end{abstract}

KEY WORDS: levels of analysis, institutional analysis, fish farms, rules-in-form

\section{Introduction}

One of the enduring challenges in the study of policy designs is creating a systematic way to organize and understand the minute elements that comprise their content. Past typologies and categorizations have been criticized for failing to provide a valid and reliable instrument for inquiry (Eulau, 1969; Froman, 1967; Lowi, 1972; Wilson, 1979). A relatively new way to categorize and relate the content of policy design was introduced by Crawford and Ostrom (1995, 2005). Crawford and Ostrom devised an institutional grammar to identify the elements within policy designs and then to create configurations of these elements toward a goal of understanding action situations. The institutional grammar helps reveal the internal structure of policies by providing a detailed depiction of what actions are allowed, permitted, and forbidden under specified conditions and often with specific sanctions for actors.

While the institutional grammar proposed by Crawford and Ostrom $(1995,2005)$ is useful for its capacity to categorize and organize policy content, it did not offer clear guidelines for implementation or empirical application (Basurto, Kingsley, 
McQueen, Smith, \& Weible, 2010; Schlüter \& Theesfeld, 2010). Basurto et al. (2010) moved Crawford and Ostrom's grammar closer to realization as a tool for analyzing policy designs by demonstrating the promise of the institutional grammar as an analytical tool in the analysis of two policies, one for transportation and the other for abortion. Basurto et al. then identified the main unresolved challenges for implementation to advance the work of scholars interested in applying the institutional grammar, for example, as a tool for the analysis of legislation (Speer, 2008) or the simulation of endogenous rule changes in agent based modeling scenarios (Smajgl, Izquierdo, \& Huigen, 2008).

In this article, we start to respond to some of the most important challenges identified by Basurto et al. (2010) by: (i) proposing an additional syntactic component to the grammar-the oBject-in order to reduce ambiguity, increase inter-coder reliability, and expand the scope of possibilities for researchers when conducting analyses relating to the institutional grammar; (ii) revising the guidelines originally proposed by Basurto et al. (2010) for coding with the grammar; and (iii) conducting an empirical analysis of Colorado State aquaculture policies to illustrate one of the new analytical possibilities for understanding cross-level linkages.

This article proceeds in two parts. The first part provides an overview of the IAD necessary to understand the conceptual origin of the institutional grammar tool (IGT). This section is largely definitional and introduces revisions to the grammar to ease the validity and reliability of its application. The second part is empirical, in which we illustrate the use of the grammar with an application to Colorado aquaculture. The conclusion discusses the future applications of the IGT, which can be applied within IAD-guided research as well as research guided by other frameworks and theories seeking understanding and explanations as to how the components of policy designs evolve over time, compare across designs, or shape and are shaped by policy processes.

\section{IAD Framework and the Institutional Grammar Tool}

The IAD framework has two main features of interest: (i) it views action situations as composed of the same set of elements or working parts, where (ii) multiple action situations exist at any one level of analysis and at various nested levels. The structure of opportunities and constraints available to actors engaged in action situations at one level are assumed to be a product of interactions between actors in situations at higher and lower levels (Ostrom, 2005, p. 58). The IAD explicitly recognizes three functional levels of analysis: the constitutional level, the collectivechoice level, and the operational level. Clearly, the actual number of levels relevant to each setting will vary. But functionally the IAD identifies the operational level, as where the day-to-day interactions take place among agents and the prescriptions they develop to affect such interactions and their outcomes. At the collective-choice level, we observe the interactions and prescriptions that affect operational activities and at the constitutional level the focus is on those prescriptions that in turn affect rules, norms, or strategies governing collective-choice arenas (Ostrom, 2005, p. 58). 
An action situation is the social space where "participants with diverse preferences interact, exchange goods and services, solve problems, dominate one another, or fight" (among the many things that individuals can do in such domains) (Ostrom, 2005, 2011, p. 14). ${ }^{1}$ The outputs of action situations ${ }^{2}$ that result in prescriptions to organize repeated interactions are defined as institutions (Ostrom, Gardner, \& Walker, 1994, p. 28). Such prescriptions can be in the form of new rules, norms, and strategies, to govern their future interactions. Thus, rules are the "shared prescriptions (must, must not, may) that are mutually understood and predictably enforced in particular situations by agents responsible for monitoring conduct and for imposing sanctions" (Ostrom, 2007, p. 23). ${ }^{3}$ Norms are the "shared prescriptions that tend to be enforced by the participants themselves through internally and externally imposed costs and inducements" (Ostrom, 2007, p. 23). Strategies represent the "regularized plans that individuals make within the structure of incentives produced by rules, norms, and expectations of the likely behavior of others in a situation affected by relevant physical and material conditions" (Ostrom, 2007, p. 23).

The IAD is a framework designed to guide inquiry particularly into the interdependencies of institutions and collective action situations. While the IAD framework can support multiple theories and models, the framework can also support multiple tools for data collection and analysis. One of these tools is the institutional grammar described by Crawford and Ostrom $(1995,2005)$. The purpose of the IGT is to unravel the minute components-analogous to genetic codes in living cells-of formal institutions, such as policies, laws, legislation, and regulations. As an IAD tool, the institutional grammar shares much of the IAD logic (Ostrom, 2005) offering refined definitions for systematically dissecting institutional statements in policy designs (see McGinnis, 2011).

For the institutional grammar tool, data are collected on "institutional statements," which are defined as "the shared linguistic constraint or opportunity that prescribes, permits, or advises actions or outcomes for actors (both individual and corporate). Institutional statements are spoken, written, or tacitly understood in a form intelligible to actors in an empirical setting" (Crawford \& Ostrom, 1995, p. 583). In the initial version of the institutional grammar tool, institutional statements were composed of five working parts: The Attribute (A), Deontic (D), aIm (I), Condition (C), and the Or else (O) (Crawford \& Ostrom, 1995, p. 584).

From these five working parts, institutional statements could then be identified as strategies, norms, and rules. A strategy contains an Attribute, an AIm, and a Condition (AIC), a norm contains, in addition, a Deontic (ADIC), and with the addition of a sanction, or an Or else, the statement becomes a rule (ADICO). Crawford and Ostrom (1995) articulate this distinction between strategies, norms, and rules within the description of the IGT to conceptually mirror the distinction applied in the IAD framework more broadly. They argued that such clear categorization of the basic elements that constitute policies is necessary for sound policy analysis, and proposed that the grammar can aid analysts in the identification of (i) actions that are required, permitted, and forbidden, (ii) the actors assigned to particular activities, (iii) the temporal and spatial boundaries in which these activities take place, and, in some cases, (iv) the punitive measures associated with noncompliance. 
However, the language associated with the strategies, norms, and rules distinction within the application of the IGT raises issues of concern as most policies (especially legislative) prescriptions may be viewed as rules. That is, all legislative prescriptions are assumed to be enforceable (i.e., have an Or else) by some agents within the policy process. We recognize the importance for the policy analyst to reach out to other legislation or legal frameworks and identify the Or else's that affect enforcement of the policy being analyzed. This search might uncover other policies that are important to the design of the policy system in question. It would also make cost-benefit or simulation/decision analysis possible. However, extending the scope of the research also increases the complexity of the design. Any observation-that is, research endeavour-requires simplification of the subject based on the research question and choice of framework and theory. Heikkila et al. (2011), for example, only code bi-state water compacts and associated documents and not the Clean Water Act or the Constitution found in the United States. This article is no different. For simplification and for the purpose of analytical clarity in our development of the grammar as a policy tool, we decided not to go beyond the four policies that comprise the aquaculture subsystem in Colorado. We categorize and refer to institutional statements in terms of the grammar components present within them.

Finally, we want to make clear that we do not view coding policies outside of their legal context as capable of providing a full dissection of a policy. For the sake of analytical clarity, we do not incorporate contextual variables yet into our analysis. It would make our understanding of the usefulness of the grammar as a policy analysis tool even more challenging. We foresee doing so at a later research step.

The next section provides a brief definition of each of the grammar components based on the original work of Crawford and Ostrom (1995) and later by Basurto et al. (2010). Table 1 provides a summary of characteristics for each of the grammar components as well as examples from coded policies.

\section{Attribute}

The Attribute is the animate agent (e.g., individual, groups of individuals, or organization(s)), that carries out the aIm. If the agent includes individuals then the Attribute might include descriptions, such as age, sex, or position (Crawford \& Ostrom, 2005, p. 141). For organizations, the Attribute might include organizational descriptions, such as organizational size (Crawford \& Ostrom, p. 141). The Attribute can be implicit or explicit in any given institutional statement (Basurto et al., 2010). For implicit attributes it is critical that the coder understand the context of the statement within the document so as to ensure that an appropriate implication is made. Further, a coder may encounter an instance in which agents are nested within larger organizations / groups, but only the former, the primary agent, is explicitly stated and the secondary agent may be inferred. For example, such an occasion is observed when an actor is a representative or employee of an organization and he/she is carrying out an action on behalf of his or her organization as a whole. In this case, it might be useful for the coder to know both the nested agent in addition to the secondary agent. 
Table 1. Summarizing Institutional Grammar Characteristics

1. Attribute Characteristic and Example from Coded Policies

- Must be an animate actor.

- May be explicit or implicit.

- Must include all relevant descriptors.

- Attribute must logically be able to perform aIm.
"A qualified fish health pathologist shall inspect all facilities annually."

"Fish health inspections shall be conducted annually [by a qualified fish health pathologist] $]^{\text {." }}$

"The fish health board shall meet annually. Descriptors = "fish health"

"The Commissioner shall enforce all rules and regulations concerning aquaculture except those which relate to fish health."

2. Deontic Characteristic and Example from Coded Policies

- The prescriptive operator of an

"The Aquaculture Board shall annually select a institutional statement that describes what is permitted, obliged, or forbidden.

- Usually explicit, but may also be implicit. chairperson."

"The Board is authorized to recommend rules to the Commissioner."

Implied Deontic $=$ may

3. AIm Characteristic and Example from Coded Policies

- Describes the goal or action of the statement, i.e., usually the verb of the statement.

- Any qualifiers of the aIm, including the identification of temporal and spatial boundaries, should be included in the Condition.
"Director of the Division may approve destruction orders."

"The aquaculture board shall annually select a chairperson and vice chairperson." aIm = "select"

Condition = "annually"

4. Condition Characteristic and Example from Coded Policies

- Includes all qualifiers of the aIm, including when, where, and how the action in the aIm is to be performed.

- Default implicit conditions is "at all times."

- Institutional statements may contain multiple conditions.
"Applications for exemption shall be submitted to the Director at least 60 days prior to any proposed stocking."

"All aquaculture facility permits must be certified [at all times]."

"Exemptions granted by the Director shall be valid unless the applicant fails to comply with the terms of the exemption or fails to submit an annual report."

Condition $1=$ ". . . unless the applicant fails to comply with the terms of the exemption..."

Condition $2=$ ". . . fails to submit an annual report..."

5. Or else Characteristic and Example from Coded Policies

- The punitive action if the directive is not followed.
"Any person that violates the provisions of this article shall be fined no less than one thousand dollars and no more than five thousand dollars."

\footnotetext{
a"[ ]" designate that statement has been implied.
} 
The best way to locate the Attribute of the statement is to identify the actor or organization which is expected to carry out the aIm, or goal or action of the statement (Crawford \& Ostrom, 2005, p. 139). In many cases, the Attribute is most clearly identifiable once one has identified the aIm of the statement. By first identifying the aIm, the coder can ensure that there is a logical relationship between the Attribute and the action being described in the aIm, that is, it is possible for the former to perform the latter.

\section{Deontic}

The Deontic is the prescriptive operator of an institutional statement that describes what ideally is permitted, obliged, or forbidden (Crawford \& Ostrom, 2005, pp. 141-149). The Deontic need not always be literally written as the words "permitted," "obliged," or "forbidden" but may also be presented in other forms, such as may, may not, must, should, must not, should not (Crawford \& Ostrom, 2005, pp. 141-149). Crawford and Ostrom (2005, p. 144) stated that Deontics must be explicit. However, Basurto et al. (2010) found that while the Deontic is most often explicitly stated, it may also be implicit. The current article follows Basurto et al.'s (2010) modification in that Deontics are allowed to be implicit under contexts where statements prescribe a command without using the words may, must, or must not. For example, the verb "required" suggests a "must" Deontic. There are also cases in which an activity is said to be "prohibited" in the policy, the implication for which would be that this activity "must not" occur. Additionally, a Deontic may be carried over, and hence implied, from a preceding institutional statement. Deontic operators can vary by prescriptive force, for example "must" represents more force than "should" (Crawford \& Ostrom, 2005, pp. 142-149). Deontics serve as useful markers for delineating institutional statements. Coders can start parsing institutional statements by looking for a Deontic and then proceeding in the following coding order: (Deontic) (aIm) (oBject) (Condition) (Or Else)/(Attribute)].

\section{Aim}

The aIm describes the goal or action of the statement that the Deontic refers to (Crawford \& Ostrom, 2005, p. 140). The aIm typically consists of all non-Deontic verbs in the statement. Any qualifiers of the aIm, including the identification of temporal and spatial boundaries relating to the action being discussed, should be included under the Condition. The interpretation of the aIm will determine the Attribute and the oBject of the statement. Additionally, the aIm may also potentially modify the Deontic. This is particularly applicable in cases where the definition of the aIm is inherently vague or when the aIm has multiple definitions and, thus, there is ambiguity about the meaning as applicable to the statement. For example, "The Director is granted authority to exempt applicants from specific pathogen testing" (Chapter 0 Regulations). In this example, "is granted" may be coded as an implicit Deontic, in which case, the Director would be the appropriate Attribute. Alterna- 
tively, if "is granted" is coded as the aIm, then the Colorado Division of Wildlife (CDoW) would be the appropriate Attribute as this is the entity performing the granting.

\section{Condition}

The Condition represents the part of the statement that modifies the aIm, often in temporal or spatial terms, but can also include descriptions of how the action identified in the aIm is to occur. As such, the condition can be thought of with the operators "when," "where," and "how" the aIm is allowed, required, or forbidden (Crawford \& Ostrom, 2005, p. 149). In other words, Conditions set the prerequisites or restrictions on the aIm. It is assumed that Conditions can be explicit or implicit (Basurto et al., 2010). When an institutional statement does not specify an explicit Condition or refer to one implicitly elsewhere, the default value is "at all times" (Crawford \& Ostrom, 2005, p. 149). An institutional statement may contain multiple conditions, so long as they do not comprise statements of their own.

\section{Or Else}

The Or else operator is the punitive action if the rule is not adhered. As was done by Basurto et al. (2010), the guidelines for coding Or else operators have been relaxed compared with the general tenets of the original grammar. For example, it is not required that the Or else operator be backed by another institutional statement for enforcement or the incentives of the monitors (Crawford \& Ostrom, 2005, p. 150). The rationale is pragmatic because each institutional statement is coded as an individual unit of observation. The Or else must be explicitly stated in order to be coded.

\section{The Introduction of the oBject}

Among the more important challenges identified by Basurto et al. (2010) for applying the grammar are (i) uncertainty in identifying the Attribute in the institutional statement; (ii) ambiguity regarding how to code statements where the Deontic is implicit rather than explicit; and (iii) difficulty in distinguishing between the aIm and the Conditions. We propose that some of these challenges can be ameliorated by the addition of one more syntactic element to the grammar: The oBject, which we describe in this section.

The oBject can be defined as the inanimate or animate part of a statement that is the receiver of the action described in the aIm and executed by the agent in the Attribute. For example, "The student wrote the paper." The oBject in this statement would be the paper which was written (aIm) by the student (Attribute). The oBject is often equivalent with the direct oBject of the sentence, but not in all cases. ${ }^{4}$

The oBject code helps avoid ambiguity when interpreting institutional statements when there is no explicit Attribute because it helps distinguish the actor (Attribute) from what the actor is acting upon (object). For example, Table 2 provides a baseline case where there is a clear agent (the student) charged with carrying out 
Table 2. Basic Illustration of the oBject Application

\begin{tabular}{|c|c|c|}
\hline & Statement & Coding \\
\hline Example One & $\begin{array}{l}\text { The student must write paper by date } \\
\text { or receive a lower final grade. }\end{array}$ & $\begin{array}{l}\mathrm{A}=\text { student } \\
\mathrm{B}=\text { paper } \\
\mathrm{D}=\text { must } \\
\mathrm{I}=\text { write } \\
\mathrm{C}=\text { by date } \\
\mathrm{O}=\text { or receive a lower final grade }\end{array}$ \\
\hline Example Two & $\begin{array}{l}\text { Paper must be written by date or } \\
\text { receive a lower final grade. }\end{array}$ & $\begin{array}{l}A=[\text { Implied }] \text { student } \\
B=\text { paper } \\
D=\text { must } \\
I=\text { be written } \\
C=\text { by date } \\
O=\text { or receive a lower final grade }\end{array}$ \\
\hline Example Three & $\begin{array}{l}\text { Student must contact the professor by } \\
\text { date or receive a lower final grade. }\end{array}$ & $\begin{array}{l}\mathrm{A}=\text { student } \\
\mathrm{B}=\text { professor } \\
\mathrm{D}=\text { must } \\
\mathrm{I}=\text { contact } \\
\mathrm{C}=\text { by date } \\
\mathrm{O}=\text { or receive a lower final grade }\end{array}$ \\
\hline Example Four & $\begin{array}{l}\text { Professor must be contacted by the student } \\
\text { by date or receive a lower final grade. }\end{array}$ & $\begin{array}{l}A=\text { student } \\
B=\text { professor } \\
D=\text { must } \\
I=\text { contact } \\
C=\text { by date } \\
O=\text { or receive a lower final grade }\end{array}$ \\
\hline
\end{tabular}

an aIm (write) on a particular oBject (paper). The second example is more challenging to code because the Attribute is implicit and the oBject takes the position of the subject of the sentence. Without the oBject, the coding would be the same for both statements; however, with its addition, potential disagreements among coders on example two would more likely be avoided. The "paper" in these examples would be the oBject because it is the element of the statement to which the Attribute and aIm apply. The clearest cases are when the Attribute is the subject of a sentence and the object is the direct object; when the sentence is passive, however, the object may be mistakenly coded as the Attribute. Including the oBject as part of the syntax reduces the ambiguity and potential for mistakes by clarifying that the receiver of the aIm is the oBject and the performer of the aIm is the Attribute. Table 2 illustrates the oBject along with the other grammar components.

The oBject code is also useful when there are two animate actors in the statement upon which the question arises-which one is the Attribute? In the second two examples in Table 2, there are two explicitly stated animate actors (student and professor) and there may be some ambiguity as to which of the two is the Attribute and which is the oBject. It is desirable to have both actors coded as individual components when conducting configuration analyses. For example, one may be curious to know how many times a particular actor appears in the document and the context in which she or he is discussed, e.g., his or her role in the action situation, the mandated, allowed, and forbidden activities relating to the role, etc. Thus, the oBject 
is useful as it allows the coder to list one of the explicitly stated actors as the Attribute and creates a new coding category in which to place the second actor.

Examples three and four in Table 2 reflect the types of cases that may be encountered by coders in which there are two animate actors. Useful strategies for coding such statements associated with using the oBject are to remember, again, that the Attribute of the statement is he/she who is expected to perform the aIm, and the oBject is the receiver of the aIm. As such, while one example is written actively and the other passively, in both cases, the student (Attribute) is to contact (aIm) the professor (oBject).

Including the oBject component additionally departs from the current grammar coding format by separating the aIm (action of the statement) from the oBject (receiver of the aIm). Here, we have limited the contents of the aIm to only include the primary action, or verb, being addressed in a particular statement. The advantage of taking this approach allows for the aIm to serve as an anchor for the statement, around which all other syntactic components can be identified. ${ }^{5}$ For example, once one knows the action that is being discussed, she or he can systematically identify who is responsible for carrying out the aIm, who or what is the receiver of the aIm, under what conditions the aIm should be performed, and the punitive sanctions associated with not performing the aIm as prescribed in the directive.

\section{Summarizing the Utility of oBject}

Given the previous discussion, it can be argued that the oBject is useful for the following reasons. First, the introduction of the oBject minimizes coding ambiguity when dealing with statements which lack explicitly stated animate Attributes and provides guidance to coders dealing with statements with apparently multiple Attributes as to which is the appropriate Attribute of the statement and which is the oBject. Thus, by minimizing coding ambiguity, the oBject code enhances the potential for inter-coder reliability. The professor-student examples in Table 2 are illustrative of this point. Second, coding the oBject as distinct from the aIm offers a clear way to delineate all other (A) (B) (D) (C) (O) components of the statement. Third, the inclusion of the oBject is also useful in the data analysis process, particularly when conducting analyses where organizing statements by syntactic component is of interest to the analyst. The utility of the oBject in this sense allows the coder more possibilities in conducting analyses where statement components are more clearly differentiated. It is more likely that the coder would choose to organize analyses and conclusions along the oBject, when considering that, in most cases, the oBject is synonymous with the direct object of the sentence and thus an integral element of the statement.

\section{Coding Guidelines}

The following general coding guidelines are a refinement of those developed by Basurto et al. (2010) to code institutional statements. Our modifications are based on the experience of coders in applying the oBject. The general purpose of these guidelines is to offer scholars using this tool a way to reliably parse institutional statements, 
a useful task to understand thoroughly and systematically the content of policies and identify theoretically and practically useful relationships between the elements presented within them (Speer, 2008), or to develop computational models where agents have the capability to endogenously create or react to different rules (Smajgl et al., 2008).

1. Identify all definitions, titles, preambles, and headings and disregard them for coding purposes. Titles and headings are first discarded because they are fairly easy to locate and rarely constitute an institutional statement of theoretical or practical interest.

2. Identify sections and subsections of the bill as initial units of observation. We call headers of sections and subsections "outline indicators." Outline indicators are titles, subheadings, capital or lowercase letters, colons, semicolons, or Roman numerals, used to separate sections from subsections and subsections from subsubsections, etc. These initial units of observation are temporary and may be divided into additional units.

3. Subdivide all initial section or subsection units from step 2 that have multiple sentences into sentence-based units of observation. If a section or subsection does not have a complete sentence ending in a period, code the entire section or subsection as one unit of observation. If there are multiple sentences in the section or subsection, code each sentence as units of observation.

4. Code the units of observation following the ABDICO syntax. The text in each unit is coded with respect to the Attribute, oBject, Deontic, aIm, Condition, and Or else. You may have more than one Attribute or aIm in the same statement. For example, if you have more than one Attribute for which all other syntactic components are identical, multiple Attributes may be included in the same statement.

Example:

"A permittee or operator shall give an invoice to the person receiving viable fish or gametes at the time of transfer of possession."

Additionally, if you have more than one aIm for which all other syntactic components are identical then multiple aIms may be included in one statement.

Example:

"The Fish Health Board shall review or initiate and consider every rule which relates to fish health." (Fish Health Board Rules)

If, however, you have more than one aIm and more than one Attribute or oBject, then the statement should be broken up so that each Attribute is distinctly assigned to each of the aIms being discussed.

Example:

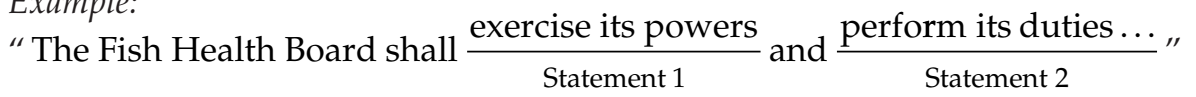

(Fish Health Board Rules) 
Further, if you have two aIms for the same Attribute or oBject but there are multiple conditions that comprise multiple institutional statements, then the statements should be broken up based on the aIm and relevant Conditions.

Example:

"Facility owners shall annually complete and submit a permit renewal application and all submissions shall be mailed by December 31 .

Statement $1: \frac{\text { Facility owners }}{\text { A }} \frac{\text { shall }}{\text { D }} \frac{\text { annually }}{\mathrm{C}} \frac{\text { complete }}{\mathrm{I}_{1}}$

and $\frac{\text { submit }}{\mathrm{I}_{2}} \frac{\text { a permit renewal application }}{\mathrm{B}}$.

Statement 2: $\frac{\text { All submissions }}{\mathrm{B}} \frac{\text { shall }}{\mathrm{D}} \frac{\text { be mailed }}{\mathrm{I}} \frac{\text { [facility owners] }}{\mathrm{A}} \frac{\text { by December } 31^{\text {st }}}{\mathrm{C}}$.

5. Code statements according to components present. In our exercise, we separated statements into AIC/ABIC, ADIC/ABDIC, and ADICO/ABDICO categories.

6. When applicable, imply components when they are not explicitly provided in the statement. In some cases, the Attribute is missing because the statement under consideration is actually an extension of the statement prior to it in the document. In this case, the coder should use the Attribute from the previous statement. In other cases, an Attribute will not be obvious, and thus an implication should be made by identifying the logical actor associated with performing the specified aIm. Sometimes in policy documents, the agent who is requiring that the action being discussed in the statement be carried out may be the Attribute. In some cases the Deontic can also be implicitly stated, as when some statements prescribe a command without using the words may, must, or must not. For the Condition component, unless stated otherwise in preceding statements, the default Condition will be "at all times," meaning that the directive is applicable in all cases unless an exception is explicitly stated.

7. Multiple coders for inter-coder reliability. As with all coding applications, each document should be coded by multiple coders to ensure that data collected through the coding process is done reliably. Coding methods should be iteratively revised until a desirable agreement percentage is met. Inter-subjective reliability should be discussed communally among the team of coders as each team will encounter difficult cases unique to the type(s) of document(s) being coded.

\section{Case Study: Colorado State Aquaculture}

We illustrate the operationalization of the grammar of institutions and its potential for policy analysis through an empirical application to all of the major laws that guide aquaculture activities in Colorado State. Aquaculture is defined as, "the farming of organisms that live in water, such as fish, shellfish, and algae" (United States Geological Survey, 1996). Aquaculture is a relevant national and state-level 
policy analysis issue given that it is one of the fastest growing food commodities (Naylor, Williams, \& Strong, 2001), and its governance is embedded in a complex regulatory framework. Aquaculture represents a private good, in that farms generate goods that are fully subtractable upon consumption (Ostrom, 2005, p. 25), but that is heavily reliant upon the availability of common pool resources (e.g., water, land, etc.). Additionally, aquaculture production poses both positive and negative externalities. Much of the regulation that has been developed around aquaculture pertains to the management of negative externalities to the natural resources upon which the industry relies.

The decrease of fish stocks in capture fisheries has served as a primary impetus to grow the U.S. aquaculture industry to meet increasing consumer demand (Boyd, 2003). Regulatory concerns relating to aquaculture include water pollution from farm effluent, competitive feed pricing, and silting issues in federal and state waters (Ackefors, Huner, \& Konikoff, 1994). The regulation of aquaculture activities occurs at multiple levels-local, state, regional, and federal —and is conducted by a number of different agencies at each geographic scale (McDaniels, Longstaff, \& Dowlatabadi, 2006, p. 426). The decentralization of regulatory responsibilities has meant that different stakeholders with varying objectives are involved at each level to decide how and when the aquaculture industry is regulated.

In the early 1990s the Colorado aquaculture industry formally requested to be incorporated into the jurisdiction of the Colorado Department of Agriculture (CDoA), thus conferring the rights and responsibilities associated with other types of agricultural activities in the state upon it. A new set of laws and regulations were created to address this jurisdictional change. This study seeks to understand some of these rules and regulations by systematically coding the institutions presented within them.

The two primary agencies charged with the regulation of aquaculture in Colorado State are the CDoA and the CDoW. The CDoA is responsible for permitting procedures relating to aquaculture and has two complementary legislative documents: the Colorado Aquaculture Act (CAA) Statute and the Rules Pertaining to the Administration and Enforcement of the Colorado Aquaculture Act, that detail the structure and responsibilities of the Aquaculture Board, provide procedural directives regarding destruction orders and all other permits and regulations present within the legislation, and articulate the fee structure assigned to different permit types.

The CDoW deals with matters of fish health and has two policy documents that deal directly with aquaculture. The first (Article VII of the Chapter 00-General Provisions) specifies prescribed fish health testing, responsibilities of the State Fish Health Pathologist, and disinfection and quarantine procedures. The second (Section 33-5.5-101 of Title 33 of the Wildlife and Parks and Regulations Rules) outlines the responsibilities of the Fish Health Board as they pertain to aquaculture.

Taken together there are four policy documents governing aquaculture activities in Colorado: the Colorado Aquaculture Act (i.e., CAA Statute), the CAA Administration and Enforcement Rules (i.e., Rules Pertaining to the Administration and 
Enforcement of the Colorado Aquaculture Act), Article VII of the Chapter 00 Regulations (herein Chapter 0), and the Fish Health Board Statute (Section 33-5.5-101 of Title 33). We coded each of these in their entirety for our analysis.

\section{Results \\ Inter-Coder Reliability}

One of the major challenges found by Basurto et al. (2010) in operationalizing the grammar was to identify reliably Attributes and Conditions from other syntactic components. To assess the effectiveness of our coding guidelines and whether the addition of a new syntactic component-the oBject-contributed to address this challenge we conducted a test of inter-coder reliability on three different policies. First, we coded 35 statements from the Colorado Aquaculture Act Administration and Enforcement Rules, constituting approximately 10 percent of the total statements coded across all four documents. ${ }^{6}$ The results from this test are included in Table 3 and show that at least 80 percent agreement was found between coders for all components. Next, we coded 10 statements from the transportation and abortion legislation coded in Basurto et al. (2010), five for each and constituting 50 percent of the total statements previously coded by them, to determine if the addition of the oBject code contributed to a higher rate of agreement between coders across statement components. Special attention was given to observing a higher agreement rate on Attributes and Conditions. The results from this test are also included in Table 3 and show that, indeed, though not entirely resolving the issues, the inclusion of the oBject code increased inter-coder reliability for both fields. ${ }^{7}$

Table 3 shows a reduction in ambiguity in the coding of all syntactic components of the grammar.

While the inter-coder reliability rates were comparably higher than those obtained by Basurto et al. (2010), our results still show most disagreement when coding Attributes, Conditions, and the oBject. Disagreements took place when coders failed to parse similar institutional statements and when coders failed to carefully assess whether certain words constituted descriptors of the oBject (in

Table 3. Comparison of Inter-Coder Reliability Test Results

\begin{tabular}{lccc}
\hline $\begin{array}{l}\text { Syntactic } \\
\text { Component }\end{array}$ & $\begin{array}{c}\text { Agreement between } \\
\text { Coders for Colorado } \\
\text { Aquaculture Policies (\%) }\end{array}$ & $\begin{array}{c}\text { Average Agreement between } \\
\text { Coders for Transportation } \\
\text { and Abortion Legislation } \\
\text { Coded with Addition } \\
\text { of oBject }\end{array}$ & $\begin{array}{c}\text { Average Agreement between } \\
\text { Coders for Transportation } \\
\text { and Abortion Legislation } \\
\text { Coded in Basurto et al. } \\
(2010)(\%)\end{array}$ \\
\hline Attribute & 86 & 90 & 82 \\
oBject & 86 & 80 & NA \\
Deontic & 97 & 90 & 89 \\
aIm & 94 & 90 & 92 \\
Condition & 80 & 80 & 67 \\
Or else & 100 & 100 & 100 \\
\hline
\end{tabular}


which case they would be included in the oBject field) or modifiers of the aIm (in which case they would need to be included in the Condition field). We acknowledge that there is always going to be some disagreement between coders due to the inherent vagueness of language. However, we find that most coding disagreements can be prevented when the coder is well acquainted with the context of the bill and the coder is able to carefully determine the aIm and from it: who carries out the aIm? (i.e., the Attribute), who or what is the receiver of the action described in the aIm? (i.e., the oBject), and how, when, where, or if the aIm is modified (i.e., the Condition). ${ }^{8}$

\section{Descriptive Results}

First, we conducted a basic frequency count for all four policy documents to determine the total number of statements within each document and categorize them by components present. Table 4 shows the results of the initial descriptive analysis.

Descriptive information such as the displayed in Table 4 is useful to identify potentially interesting trends and tendencies in the data. In our case, for instance, it draws attention to the fact that in comparison with all other policies, the CAA Administration and Enforcement Rules contain a proportionally high number of institutional statements containing only Attributes, Aims, and Conditions (30 percent); that is, statements without a Deontic (the prescriptive operator that describes what ideally is permitted, obliged, or forbidden) and Or else (the punitive sanction associated with noncompliance with the policy directive). For example, "Amendments to these rules are proposed for adoption by the Commissioner of the Colorado Department of Agriculture" (CAA Rules). This finding might warrant further inquiry for the analyst about the particulars of the institutional statements that conform to it. Among others, a potentially interesting question for the analyst to pursue might be: What are the reasons an agency devoted to administration contains relatively few guidelines describing what is permitted, obliged, or forbidden?

Further, Table 4 indicates that there is only one institutional statement containing all grammar components in the sample of policy documents (CAA Statute). This finding is consistent with Basurto et al.'s (2010) study. However, when applying the grammar to the study of formal policies the lack of Or else in a statement does not suffice to conclude that no punitive measures are associated with noncompliance. Given the nested quality of formal policies, higher levels of government or different agencies authorized by other policies are in a position to apply punitive measures against those who fail to comply with policy directives.

Next, for the four policy documents we also conducted a frequency count to determine the three Attributes, oBjects, and Deontics most frequently occurring within each, the results of which are contained in Table 4. Conditions were not included within this analysis because this field contained a lot of information that varied significantly between statements. Thus, information within this field was not 


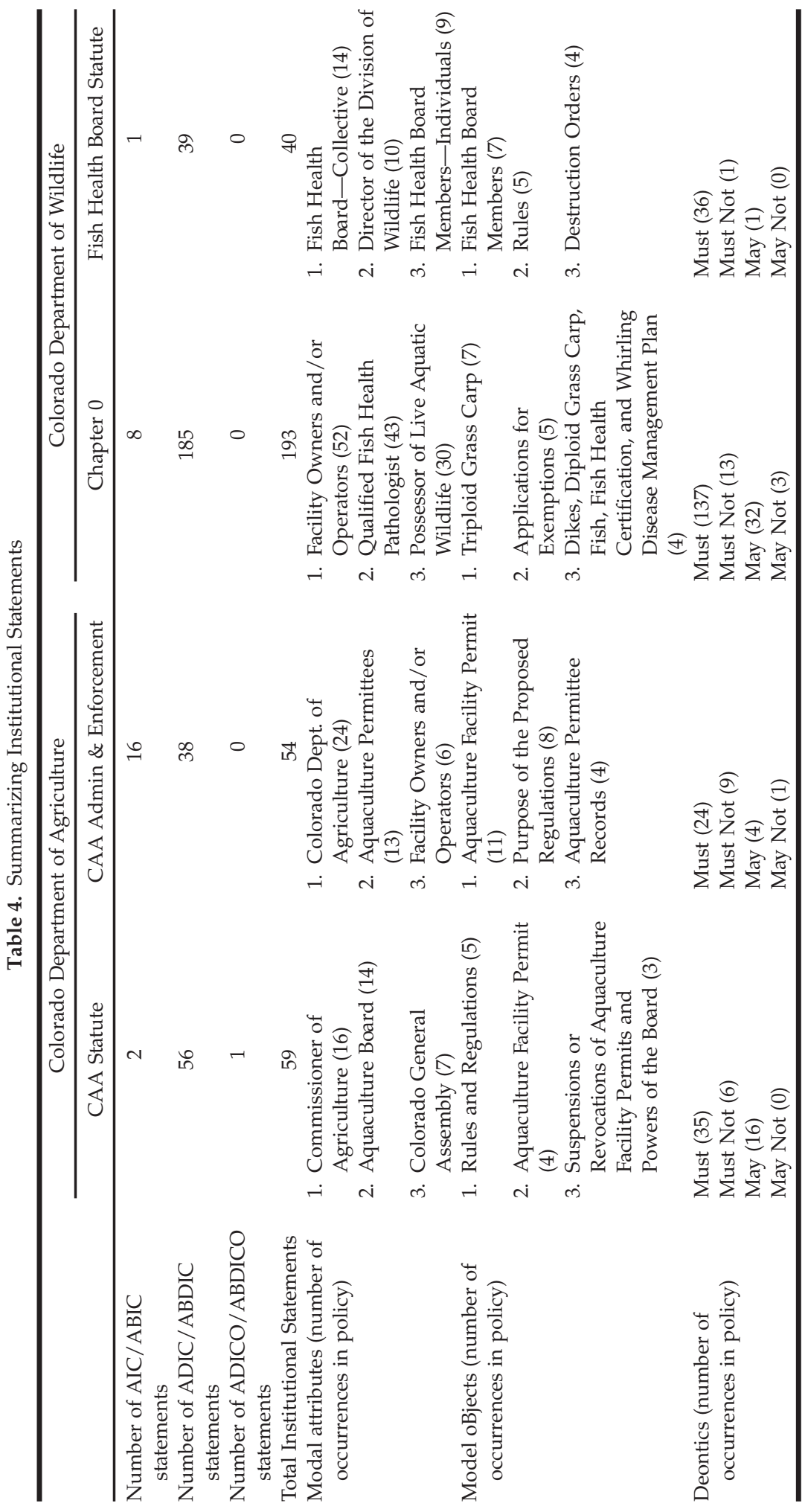


amenable to systematic compartmentalization. Or elses were not included due to the rarity of their occurrence in the policies coded. A frequency count of aIms was conducted, but due to the high amount of variability between statements, the results are not presented here.

A preliminary overview of Table 4 allows one to begin to compare and contrast the scope of the four policies. With regard to policy actors, the Colorado Aquaculture Act, for example, depicts actors such as the Colorado Aquaculture Board and the Commissioner of Agriculture as having primary regulatory and implementation authority. As these two documents originate from the same agency, the Colorado Administration and Enforcement Rules were also likely to include the CDoA but additionally include aquaculture facility owners and operators. A basic comparison of Attributes, thus, highlights the primary actors involved in the implementation process and hints at the relative authority that actors have in making decisions that shape aquaculture action situations. Also observable in Table 4 is insight into the intent of the actions shaped by the policies through the modal OBjects. oBjects illuminate the focal points of regulatory processes and procedures with which policy actors are associated.

Where many oBjects are prescribed to single actors, an implication may be made that his or her scope of activities constitute a wide range, thus signaling more involvement in the aquaculture arena. The converse may also be implied. For example, the oBjects related to the Commissioner of Agriculture relate primarily to dealing with aquaculture rules and permit requirements, thus implying that this actor's scope of activities involves a higher level management of aquaculture related issues limited to a few specific areas. This contrasts with facility owners and operators who are associated with a wide range of oBjects from fish health testing, to administrative procedures, to facility management techniques. This comparison is assumed to be a generally accurate representation regarding the involvement of these modal actors in the aquaculture industry, for while aquaculture constitutes only one of the agricultural areas with which the Commissioner deals, aquaculture for a facility owner likely is his or her primary daily activity.

In summarizing the modal Attributes, oBjects, and Deontics within each policy document in this manner, one may begin to discern how individual components from institutional statements cumulatively structure action situations described within policies (Ostrom, 2005, p. 175). Within each policy document one may observe who are the primary participants, and various characteristics regarding them, including, for example, the activities associated with them and the control that their respective positions afford. The data may then be used to derive empirically testable questions. Examples of questions include: To what extent do Deontics signal the discretionary authority of modal actors? How do Attributes and oBjects relate to one another and what do these relationships imply about the scope of authority and/or responsibility of modal Attributes? Because the information gleaned from such tables is limited, however, one may conduct a configuration analysis to uncover additionally meaningful trends and tendencies. 


\section{Configuration Analysis}

To illustrate the analytic potential of the institutional grammar Figure 1 shows (i) how political actors and organizations are linked across levels of analysis around shared processes (Kiser \& Ostrom, 2000); and (ii) maps these processes, through the identification and configuration of components, in order to understand how they are intended to guide the behavior of actors through outlining "strategic options and role expectations" (Kiser \& Ostrom, 2000, p. 6; Koontz \& Hardy, 2009). As a result the analyst may observe (i) how different coded components are linked to animate a particular Attribute's behavior regarding a prescribed political process; (ii) how Attributes themselves are linked in relation to policy directed processes, in terms of both their individual and organizational interactions; and (iii) what are the policy procedures associated with modal Attributes in relation to levels of analysis? A discussion of each of these in relation to Figure 1 is provided below.

Mapping How Different Components of the Institutional Grammar Structure Opportunities and Constraints to Different Policy Actors (i.e., Attributes). In order to determine how policy related actors are expected to act, one must first know who the primary actors are whose behavior policies are intended to shape. In Figure 1, the modal Attribute from each of the policies examined is represented in the left hand column. The Commissioner of Agriculture is from the Colorado Aquaculture Act Statute (CDoA), the CDoA is from the Colorado Aquaculture Act Administration and Enforcement Rules (CDoA), the Fish Health Board is from the Fish Health Board Rules (CDoW), and Facility Owners and/or Operators are from the Chapter 0 Regulations $(\mathrm{CDoW})$. Each of these Attributes is discussed in relation to the oBject most frequently occurring across the two Attributes in the two documents for each of the respective agencies. For instance, the oBject "Aquaculture Permits" is implemented by two different actors or Attributes: the CDoA and the Commissioner of Agriculture. These Attributes determine the total possible aIms that must, must not, or may be performed in relation to the oBject. Following this example, on the one hand, the CDoA must not deny an aquaculture permit for local and nonthreatening species, but may also deny or limit an application for an aquaculture permit or its renewal, or must expire facility permits on December 31 of each year, to name just three of seven different institutional arrangements that the $\mathrm{CDoA}$ is capable of as related to aquaculture permits. On the other hand, the Commissioner of Agriculture can also issue directives related to the same object, "aquaculture permits" i.e., may withhold, deny, or revoke aquaculture permits, or must provide for the issuance of permits or must establish permit fees. Note that it is through this mapping that one can understand how statement components relate to one another in structuring the opportunities, constraints, apparent contradictions, and potential for conflict among modal actors vis-à-vis policy directives.

Uncovering Institutional Diversity. By anchoring Attributes and aIms around a shared oBject as done in Figure 1, we demonstrate how one oBject may formally appear in a variety of action situations as prescribed by the various policies and 


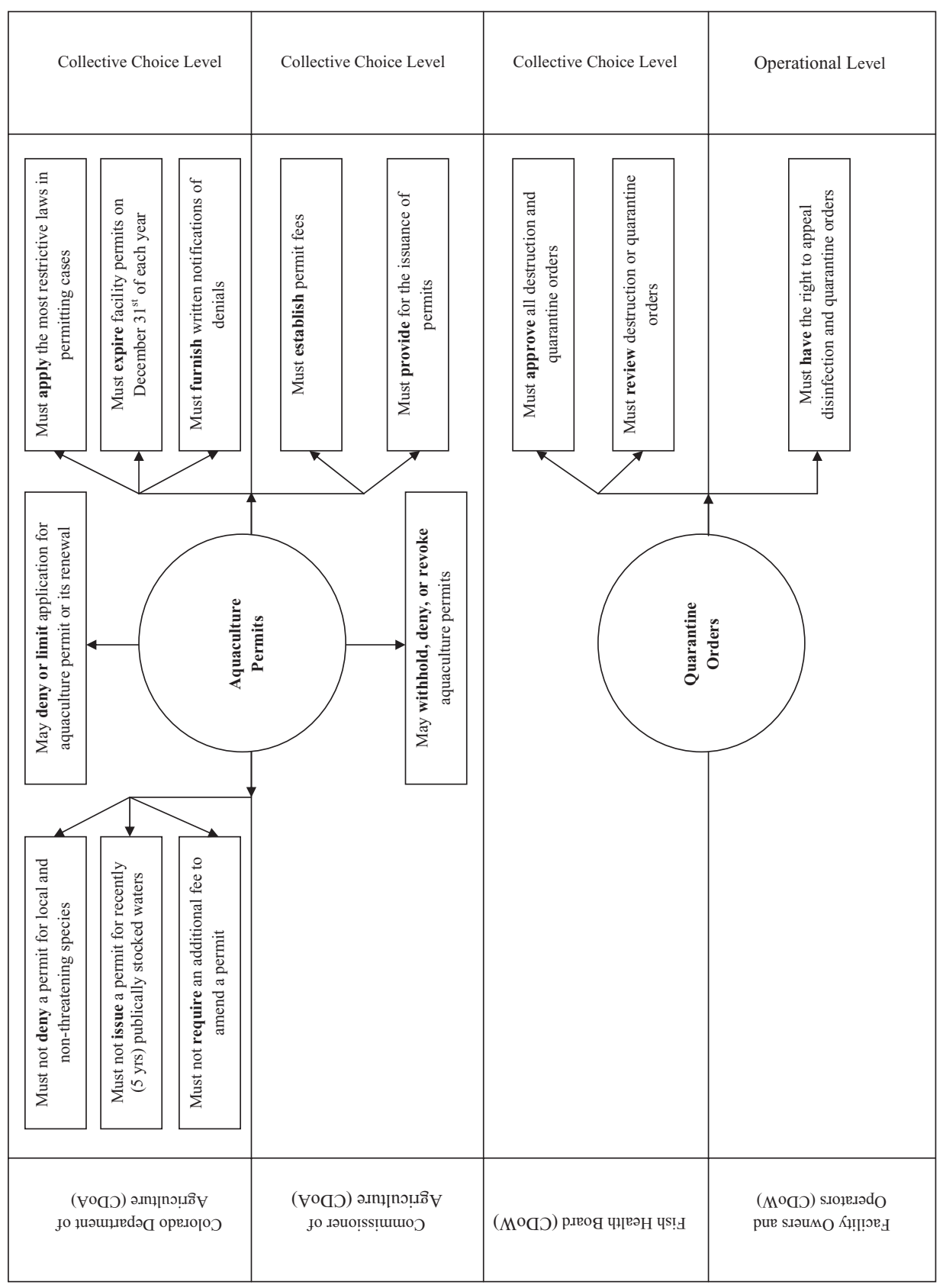

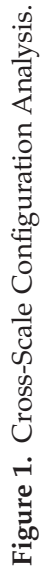


involving multiple actors, organizations, and processes; that is, the various opportunities and constraints available to actors via prescribed processes and related prescriptive operators in relation to a particular oBject. Such a depiction segues to a host of analytical possibilities. For example, one can explore the networks of actors associated with an oBject, or how different action situations might be linked (McGinnis, 2011). Additionally, one may also consider how actors' individual and organizational affiliations shape how they interact with the relevant oBject (Bushouse, 2011). For example, in the bottom half of Figure 1, the oBject depicted is "Quarantine Orders." The two actors portrayed in relation to quarantine orders are the Fish Health Board, the members of which are formally opportuned with "Approving destruction and quarantine orders," and Facility Owners and/or Operator who complementarily "Have the right to appeal all disinfection and quarantine orders." Not only does this demonstrate the formal opportunities available to each of the actors relating to a shared oBject, but the ways in which they may interact in an action situation concerning quarantine orders.

Linking Attributes through Different Levels of Analysis. Mapping policy documents as in Figure 1 might be theoretically useful to an analyst interested in determining formal actors' constraints and opportunities at different levels of analysis. As Kiser and Ostrom (2000, p. 6) write: "At each level individual and collective choice is constrained to some range of strategic options. The point of this demarcation of levels is to highlight some fundamental similarities among political processes at different levels of analysis. At each level, actors confront an action situation with strategic options and role expectations as defined at higher levels, and the choice of actors at one level jointly produce patterns of interactions and outcomes."

The level of analysis at which the Attributes' prescribed responsibilities are understood to occur is shown to the right hand side of the configuration analysis for each actor, and is displayed as either being the "Collective Choice Level" or "Operational Choice Level." The decision regarding which level to place the respective Attributes at is determined by observing the types of aIms associated with each actor. This discussion is not to imply that actors only act at one level of decision making, but rather to showcase how their roles and responsibilities at different levels are formalized within policy designs. For example, most of the tasks associated with the CDoA and the Commissioner of Agriculture represent activities that would shape the structure of activities dealing with aquaculture permits at an operational level; the former assigned such duties as "issuing permits" and "applying the most restrictive state/federal laws for permitting," and the latter with "establishing permit fees" and "providing for the issuance of permits." Since these activities can be understood to shape rules that structure activities at the operational level, each of these Attributes are assumed to work primarily at a collective choice level. Similarly, the Fish Health Board is also charged with tasks associated with the collective choice level such as "approving all destruction and quarantine orders" and "reviewing destruction or quarantine regulations." Conversely, facility owners are evidently not in a position to determine the structure of administering quarantine orders, however they do "have the 
right to appeal" them. As such, it is determined that facility owners affect quarantine orders within action situations at the operation level.

\section{Conclusion}

One of the biggest theoretical and practical challenges facing public policy scholars is developing a systematic understanding of formal institutional arrangements, usually referred to as written policies. The application of the institutional grammar provided herein illustrates a systematic approach to identifying the basic elements of written policies and features an illustrative analysis to demonstrate how minute elements of policies may be aggregated and configured to uncover useful practical and theoretical relationships. From the analysis of each policy document, we gained insight into the various ways that these policies seek to structure the activities of aquaculture participants in Colorado State. A next step is to arrange coded information in terms of specific types of action situations that statements are meant to affect. That is, how statements articulate the comparable positions of these participants, how individuals arrive at these positions, and what they may or may not do once they are there (Koontz \& Hardy, 2009).

Clearly, the IAD framework has a dual emphasis on formal and informal institutions. Our work illustrated here on the IGT only provides insights on the structure of formal institutions, such as other institutional analysis featured in this volume and beyond (Heikkila et al., 2011; Schlager \& Heikkila, 2009). For example, as part of their study to examine cross-scale institutional linkages, Heikkila et al. (2011) code bi-state water compacts using an IAD guided coding framework. Other applications of the IAD only focus on informal institutions (Basurto, 2005). Logically, the next steps ought to focus on both formal and informal institutions and the relationship between them in shaping the behavior of individuals. Such an application would necessitate that the IGT be applied in concert with other forms of data collection that can capture informal institutions. Interviews in which participants are asked to juxtapose their daily activities with those presented within formal institutions, for example, could be used toward this aim.

One way this article contributes to the emerging study of the application of Crawford and Ostrom's institutional grammar is by introducing the oBject. The oBject is incorporated into the institutional grammar and coding guidelines are clarified to address challenges posed by Basurto et al. (2010, p. 14). The first of these challenges deals with conceptually defining the Attribute. In past applications the Attribute was defined as "to whom or what the institutional statement applies." With the inclusion of the oBject a clear distinction is made regarding who is expected to carry out the aIm (Attribute) and who or what is expected to receive the aIm (oBject). In addition, by limiting the definition of the aIm to only include the non-Deontic verb of the statement, ambiguity concerning the distinction between the aIm and the Condition is reduced. In the revised definition, one or few verbs should be included in the aIm, and all modifiers should be included in the Condition field.

Scholars need not restrict the application of the IGT to the logic of the IAD, as was done in this article. The institutional grammar offers a methodological tool for 
collecting data to systematically understand the content of policy design. The theoretical analysis and interpretation of the resulting data may be informed by a wide variety of policy process models, theories, and/or frameworks. For example, scholars applying the social construction framework may find it most useful to configure the coded data around sanctions, or Or else components, in relation to different actor categories presented in the policy document to understand how benefits and burdens are distributed across different policies (Mondou \& Montpetit, 2010; Schneider \& Ingram, 1997). Further, data could be configured to support the application of agent-based models that seek to understand how policies shape actors' individual and aggregated decision-making behaviors (Janssen, 2005; Smajgl et al., 2008). The advocacy coalition framework predicts that policies are but the translation of beliefs; thus, this grammar provides a means for linking coalition beliefs to institutions (Sabatier \& Jenkins-Smith, 1993). Moreover, the IGT could aid any theory of policy change to understand how institutions evolve over time. In IAD guided research, the IGT could be used to assess the extent of congruence between formal and informal institutions thereby providing insight into effective monitoring and enforcement as well as effective implementation.

For those who do wish to study coded data from the perspective of the IAD framework, future research using the IGT should elaborate upon theoretical issues that we address in our analysis. Namely, this involves exploring the two analytical approaches we proffer to develop theoretically motivated questions. To reiterate, these two approaches, include: (i) mapping political processes or procedures associated with modal Attributes in relation to levels of analysis; and (ii) demonstrating how different coded components are linked to animate a particular Attribute's behavior regarding a prescribed political process.

As mentioned above, future research could consider supplementing policy coding with other forms of data collection methods, such as interviews or a questionnaire, to test empirical questions derived from relationships identified in coded data; for example, interviewing policy relevant actors in the action situations to understand how they are interpreting policy directives and, further, which statement components are most influential in shaping their interpretation. Similarly, researchers may apply the institutional grammar to generate data for more sophisticated analysis. For example, network analysis techniques could be used to identify the centrality of certain attributes, density of institutions in policies, or the links between different policies over time or across policy subsystems.

We want to thank a reviewer for pointing out that future attention and study needs to be given to the issue of rules of permission; that is, "constituting rules" found in the original grammar publications (Crawford \& Ostrom, 1995). Take a previous example: "Amendments to these rules are proposed for adoption by the Commissioner of the Colorado Department of Agriculture." This is an example of a "constituting" or "authority granting" rule because there is an implied "may" in the institutional statement that the Commissioner may propose amendments to the rules. If amendments not proposed by the Commissioner are invalid then the full coding of the statement is: commissioner may propose amendments Or else amendments are invalid. These kinds of authority granting rules are really impor- 
tant in understanding positions and how policies can change. This is very different than a strategy that emerged where it was decided that it was best for the Commissioner to propose amendments but it would not be invalid if someone else were to propose amendments instead. Since much of policy design is establishing who has authority to do what, these rules are really important. Reviewing the coding on these permission rules with a clearer understanding of constituting authority will be an important revision.

A similar element of institutional grammar tool that is currently overlooked when coding the oBject is the distinction between actions and outcomes. For example, some oBjects will serve to require attributes to take certain actions (e.g., install scrubbers) and others will require certain outcomes (e.g., setting pollution levels). We consider oBjects as actions and outcomes, at the least, an area worthy of exploration. Nonetheless, increasing the nuances and specificity of the coding framework is an important goal as long as extra effort devoted to coding is exceeded by the subsequent gains in knowledge. No framework, theory, or tool can describe and explain everything.

Understanding the content and interactions of policy designs has puzzled researchers for decades. The challenge is that policy designs are composed of elements traced from prior politics with nontrivial interdependencies resulting in various outputs and outcomes; that is, policy designs can be thought of as analogous to complex systems (Simon, 1996). In complex systems, boundaries and scales are artificial, requiring both simplifying assumptions and cross-scale theorizing. The initial move in studying complex systems is to identify and define the elementary elements of the system both reliably and validly, for faulty description precludes explanation. The next move is to configure those elements to present a simplified depiction of the system and then to answer research questions, to test hypotheses, or both. Scholars seeking to provide more complete depictions of the ways in which elements of different sets of formal institutions governing a particular activity are related may do so by coding policies nested across levels of government. For example, by coding and comparatively analyzing related sets of national and state level of policies.

What the grammar helps to illustrate is the overall interrelationship between who (Attribute), is allowed, permitted, forbidden (Deontics) to perform what actions (aIm), and under what Conditions. That is, it helps to uncover the underlying structure of a long body of institutional statements, often called policies. The conjecture is that understanding what this structure looks like might in fact help a policy analyst understand, for instance, what are the different levels at which the policies operate, who are the main participants involved, and where most of the decisionmaking power (actions under conditions) is located, which in turn might determine the overall degree of "enforceability" of the set of institutional statements being analyzed. While not attempted in this article, the end goal is to link the elements and the configuration of elements to a broader system of actors, physical and material conditions, community characteristics, and, especially, the political processes that created the policy design and the political processes that proceed from the policy design. 
Saba Siddiki is a PhD Candidate at the School of Public Affairs University of Colorado Denver.

Christopher M. Weible is an assistant professor at the School of Public Affairs University of Colorado Denver.

Xavier Basurto is an assistant professor at the Duke Marine Lab of the Nicholas School of the Environment, Duke University.

John Calanni is a PhD Candidate at the School of Public Affairs University of Colorado Denver.

\section{Notes}

1. The social space itself is conceptualized as an action situation, and the action situation together with participants constitute an action arena. For the purposes of this article, it suffices to only use the term action situation. For a more detailed description and nuanced conceptualization of each term-as well as the IAD as a whole-see Ostrom $(2005,2007)$.

2. All action situations are conformed by the same clusters of variables, and thus what results from the immense number of ways the clusters of variables combine with each other is the large variety of action situations that we observe in the world every day. Describing the different working parts of an action situation is beyond the scope of this article. Please refer to Ostrom $(2005,2007)$ for a detailed description of each of them.

3. We make this remark because under the IAD tradition, there is a clear conceptual distinction among frameworks, theories, and models (Ostrom, 2007, p. 25). But also because in contrast to the definition of rules, norms, and strategies provided here, the one provided under the grammar of institutions is much narrower in scope to fit the micro institutional scale under which the grammar operates.

4. Mondou and Montpetit (2010) also use an oBject in their use of prepositional analysis.

5. The aIm also serves as the basis to link the grammar to the rule typology proposed by Ostrom (2005), which in turn opens new analytical possibilities.

6. The coding was conducted by two PhD students, John Calanni and Saba Siddiki, who were trained in the institutional grammar tool and who had experience with aquaculture policies in the United States in the aquaculture partnership project (Calanni, Weible, \& Leach, 2010). Coded items by both students were discussed with assistant professors Xavier Basurto and Chris Weible. Prior to the inter-coder reliability test, the four members of the research team engaged in three months of preliminary, informal inter-coder reliability exercises involving sample institutional statements from the Colorado aquaculture policies. The goal of the preliminary-round coding was to train members of the research team in the institutional grammar tool, to become familiar with Colorado aquaculture, and to clarify aspects of the coding framework. Inter-coder reliability was calculated according to percentage agreement on coded items between coders Calanni and Siddiki (Lombard, Snyder-Duch, \& Campanella Bracken, 2002). While this method has been criticized for reasons such as its lack of ability to account for chance agreement between coders as well as the failure to reflect degrees of agreement (Lombard et al., 2002), we argue that such criticisms are not valid when applying the institutional grammar tool. Unlike coding processes involved in other types of content analysis, the original description of the IGT and subsequent revisions offer detailed instructions on how legislative documents should be deliberately coded to reflect different a priori identified components of institutional statements (Attribute, oBject, Deontic, etc.). Components generally contain a few words or short phrases that clearly belong, or not, to certain categories. As such, the possibility of identifying coded items correctly by chance is reduced, as is the potential for only partially populating a coding category with the correct information.

7. The recoding of the abortion and transportation policies from Basurto et al. (2010) was done by Xavier Basurto and Saba Siddiki.

8. It is our position that it will be up to individual teams of coders to clarify coding guidelines regarding each of the statement components to suit their coding needs and objectives to achieve a suitable agreement percentage for their purposes. Our primary objective in conducting an inter-coder reliability test was to ensure that our data were collected based upon reliable methods and guidelines. Data collection was completed in accordance with coding rules and strategies previously articulated by the other scholars and further developed based upon our own coding experiences (Basurto et al., 2010; 
Crawford \& Ostrom, 1995). The selection of coding criteria is not meant to express normative evaluations and/or biases of the data by the authors. Such normative considerations arise in the interpretation of the data and reflect the epistemological biases of the researchers.

\section{References}

Ackefors, Hans, Jay V. Huner, and Mark Konikoff. 1994. Introduction to the General Principles of Aquaculture. New York: The Haworth Press, Inc.

Basurto, Xavier. 2005. "How Locally Designed Access and Use Controls Can Prevent the Tragedy of the Commons in a Mexican Small-Scale Fishing Community." Society and Natural Resources 18: 643-59.

Basurto, Xavier, Gordon Kingsley, Kelly McQueen, Mshadoni Smith, and Christopher M. Weible. 2010. “A Systematic Approach to Institutional Analysis: Applying Crawford and Ostrom's Grammatical Syntax." Political Research Quarterly 63: 523-37.

Boyd, Claude E. 2003. "Guidelines for Aquaculture Effluent Management at the Farm Level." Aquaculture 226: 101-12.

Bushouse, Brenda K. 2011. "Governance Structures: Using IAD to Understand Variation in Service Delivery for Club Goods with Information Asymmetry." Policy Studies Journal 39 (1): 99-113.

Calanni, John, Christopher M. Weible, and William, D. Leach. 2010. “Explaining Coordination Networks in Collaborative Partnership." Paper presented at the Midwest Political Science Association Meeting, April 22-25, Chicago, IL.

Crawford, Sue E.S., and Elinor Ostrom. 1995. "A Grammar of Institutions." American Political Science Review 89 (3): 582-600.

Crawford, Sue, and Elinor Ostrom. 2005. "A Grammar of Institutions." In Understanding Institutional Diversity, ed. Elinor Ostrom. Princeton, NJ: Princeton University Press.

Eulau, Heinz. 1969. Behavioralism in Political Science. New York: Atherton Press.

Froman, Lewis A. 1967. The Congressional Process: Strategies, Rules, and Procedures. Boston: Little, Brown.

Heikkila, Tanya, Edella Schlager, and Mark W. Davis. 2011. “The Role of Cross-Scale Institutional Linkages in Common Pool Resource Management: Assessing Interstate River Compacts." Policy Studies Journal 39 (1): 115-39.

Janssen, Marco. 2005. Agent-Based Modelling. Entry prepared for the Internet Encyclopaedia of Ecological Economics. International Society for Ecological Economics.

Kiser, Larry, and Elinor Ostrom. 2000. "Three Worlds of Action: A Metatheoretical Synthesis of Institutional Approaches." In Polycentric Games and Institutions: Readings from the Workshop in Political Theory and Policy Analysis, ed. Michael D. McGinnis. Ann Arbor: University of Michigan Press.

Koontz, Tomas M., and Scott D. Hardy. 2009. "Rules for Collaboration: Institutional Analysis of Group Membership and Levels of Action in Watershed Partnerships." Policy Studies Journal 37 (3): 393-414.

Lombard, Matthew, Jennifer Snyder-Duch, and Cheryl Campanella Bracken. 2002. "Content Analysis in Mass Communication: Assessment and Reporting of Intercoder Reliability." Human Communication Research 28 (4): 587-604.

Lowi, Theodore J. 1972. "Four Systems of Policy, Politics, and Choice." Public Administration Review 32 (4): 298-310.

McDaniels, Timothy, Holly Longstaff, and Hadi Dowlatabadi. 2006. “A Value-Based Framework for Risk Management Decisions Involving Multiple Scales: A Salmon Aquaculture Example." Environmental Science and Policy 9: 423-38.

McGinnis, Michael D. 2011. "Networks of Adjacent Action Situations in Polycentric Governance." Policy Studies Journal 39 (1): 45-72.

Mondou, Matthieu, and Eric Montpetit. 2010. “Avoiding Degenerative Politics: Poverty Policy Designs and Styles in Newfoundland and Quebec." Policy Studies Journal 38 (4): 703-22.

Naylor, Rosamond L., Susan L. Williams, and Donald R. Strong. 2001. “Aquaculture-A Gateway for Exotic Species." Science 294 (5547): 1655-6.

Ostrom, Elinor. 2005. Understanding Institutional Diversity. Princeton, NJ: Princeton University Press. 
2007. "Institutional Rational Choice: An Assessment of the Institutional Analysis and Development Framework." In Theories of the Policy Process, ed. Paul Sabatier. Boulder, CO: Westview Press.

- 2011. "Background on the Institutional Analysis and Development Framework." Policy Studies Journal 39 (1): 1-21.

Ostrom, Elinor, Roy Gardner, and James Walker. 1994. Rules, Games, and Common-Pool Resources. Ann Arbor: University of Michigan Press.

Sabatier, Paul A., and Hank Jenkins-Smith. 1993. Policy Change and Learning: An Advocacy Coalition Approach. Boulder, CO: Westview Press.

Schlager, Edella, and Tanya Heikkila. 2009. "Resolving Water Conflicts: A Comparative Analysis of Interstate River Compacts." Policy Studies Journal 37 (3): 367-92.

Schlüter, Achim, and Insa Theesfeld. 2010. "The Grammar of Institutions: The Challenge of Distinguishing between Strategies, Norms, and Rules." Rationality and Society 22 (4): 445-75.

Schneider, Ann, and Helen Ingram. 1997. Policy Design for Democracy. Lawrence: University Press of Kansas.

Simon, Herbert. 1996. The Sciences of the Artificial. Cambridge, MA: MIT Press.

Smajgl, Alex, Luis R. Izquierdo, and Marco Huigen. 2008. "Modeling Endogenous Rule Changes in an Institutional Context: The ADICO Sequence." Advances in Complex Systems 11 (2): 199-215.

Speer, Johanna. 2008. Why Participatory Service Governance Remains an Empty Promise: A Application of the Grammar of Institutions to Guatemala's Development Council Law. Working Paper presented at the Mini-conference of the Seminar on Institutional Analysis and Development (Y 673) at the Workshop in Political Theory and Policy Analysis, Bloomington, Indiana, January 12, 2008.

United States Geological Survey. Last Modified 1996. Glossary of Water Use Terminology. http:// water.usgs.gov/watuse/wuglossary.html Accessed June 15, 2009.

Wilson, James Q. 1979. American Government: Institutions and Policies. Lexington, MA: D.C. Health. 